\section{Human erythroid differentiation requires VDAC1-mediated mitochondrial clearance}

\author{
Martina Moras, ${ }^{1,2,3}$ Claude Hattab, ${ }^{1,2,3}$ Pedro Gonzalez-Menendez, ${ }^{3,4}$ Claudio M. \\ Fader, ${ }^{5,6}$ Michael Dussiot, ${ }^{3,7}$ Jerome Larghero, ${ }^{8}$ Caroline Le Van Kim,, ${ }^{1,2,3}$ \\ Sandrina Kinet,, ${ }^{3,4}$ Naomi Taylor, ${ }^{3,4,9}$ Sophie D. Lefevre ${ }^{1,2,3 \#}$ and Mariano A. \\ Ostuni ${ }^{1,2,3 \#}$
}

${ }^{1}$ Université de Paris, UMR_S1134, BIGR, Inserm, Paris, France; ${ }^{2}$ Institut National de Transfusion Sanguine, Paris, France; ${ }^{3}$ Laboratoire d'Excellence GR-Ex, Paris, France; ${ }^{4}$ Institut de Génétique Moléculaire de Montpellier, Université de Montpellier, CNRS, Montpellier, France; ${ }^{5}$ Laboratorio de Biología Celular y Molecular, Instituto de Histología y Embriología (IHEM), Universidad Nacional de Cuyo, CONICET, Mendoza, Argentina; ${ }^{6}$ Facultad de Odontología, Universidad Nacional de Cuyo, Mendoza, Argentina; 'Université de Paris, UMR_S1163, Laboratory of Cellular and Molecular Mechanisms of Hematological Disorders and Therapeutical Implication, INSERM, Paris, France; ${ }^{8} \mathrm{AP}$-HP, Hôpital Saint-Louis, Unité de Thérapie Cellulaire, Paris, France and ${ }^{9}$ Pediatric Oncology Branch, Center for Cancer Research (CCR), National Cancer Institute (NCl), National Institutes of Health (NIH), Bethesda, MD, USA

"SDL and MAO contributed equally as co-senior authors

\section{ABSTRACT}

$\prod$ ryter rythroblast maturation in mammals is dependent on organelle clearance throughout terminal erythropoiesis. We studied the role of the outer mitochondrial membrane protein voltage-dependent anion channel-1 (VDAC1) in human terminal erythropoiesis. We show that short hairpin (shRNA)-mediated downregulation of VDAC1 accelerates erythroblast maturation. Thereafter, erythroblasts are blocked at the orthochromatic stage, exhibiting a significant decreased level of enucleation, concomitant with an increased cell death. We demonstrate that mitochondria clearance starts at the transition from basophilic to polychromatic erythroblast, and that VDAC1 downregulation induces the mitochondrial retention. In damaged mitochondria from non-erythroid cells, VDAC1 was identified as a target for Parkin-mediated ubiquitination to recruit the phagophore. Here, we showed that VDAC1 is involved in phagophore's membrane recruitment regulating selective mitophagy of still functional mitochondria from human erythroblasts. These findings demonstrate for the first time a crucial role for VDAC1 in human erythroblast terminal differentiation, regulating mitochondria clearance.

\section{Introduction}

Mature erythrocytes result from a finely regulated process called erythropoiesis, producing two million erythrocytes every second in healthy human adults. ${ }^{1}$ In mammals, during the late stages of terminal erythroid differentiation, erythroblasts expel their nuclei and lose their organelles including the Golgi apparatus, endoplasmic reticulum (ER), ribosomes and mitochondria. ${ }^{2}$ Organelles can be eliminated by the general process of macro-autophagy. This process is initiated by the formation of a double membrane structure called phagophore, engulfing cytoplasmic material to form the autophagosome which fuses with a lysosome for degradation. ${ }^{3}$ Alternatively, mitochondria can be eliminated by a selective autophagic process called mitophagy, in which outer mitochondrial membrane (OMM) proteins act as signals to recruit the phagophore membrane. ${ }^{4}$ In erythroid progenitors, much work has shown that autophagy is essential for the organelle removal that occurs during reticulocyte maturation. ${ }^{5,6}$ Notably though, the majority of these studies were performed in human progenitors that had already reached the reticulocyte stage of differentiation or in genetic mouse models that
Ferrata Storti Foundation

Haematologica 2022

Volume 107(1):167-177

\section{Correspondence: \\ MARIANO A. OSTUNI \\ mariano.ostuni@inserm.fr \\ SOPHIE D. LEFEVRE \\ sophie.lefevre@inserm.fr \\ Received: April 29, 2020. \\ Accepted: December 21, 2020. \\ Pre-published: January 7, 2021.}

https://doi.org/10.3324/haematol.2020.257121

(C)2022 Ferrata Storti Foundation

Material published in Haematologica is covered by copyright. All rights are reserved to the Ferrata Storti Foundation. Use of published material is allowed under the following terms and conditions:

https://creativecommons.org/licenses/by-nc/4.0/legalcode. Copies of published material are allowed for personal or internal use. Sharing published material for non-commercial purposes is subject to the following conditions:

https://creativecommons.org/licenses/by-nc/4.0/legalcode, sect. 3. Reproducing and sharing published material for commercial purposes is not allowed without permission in writing from the publisher. 
were deleted for different proteins involved in phagophore formation (i.e., Ulk1, Atg4, Atg7). In these latter models, the importance of autophagy in murine erythroid differentiation was demonstrated by the finding that the delayed clearance of mitochondria and ribosomes is associated with anemia. ${ }^{7-11}$

Mitophagy results from the binding of adaptors to LC3 (microtubule-associated protein 1 light channel $3 \mathrm{~B}$, also known as ATG8) within the growing autophagosome. Once associated with phosphatidyl ethanolamine (PE), LC3-II (PE-conjugated LC3) enables the elongation and maturation of the phagophore that is recruited to ubiquitin-covered mitochondria through interaction with the LIR (LC3-interacting region) motif of mitophagy adaptors. An autophagosome is then built around mitochondria that are finally degraded following autophagosomelysosome fusion. Mitophagy can also occur in an ubiquitin-independent manner following accumulation of OMM proteins such as NIX/BNIP3L, ${ }^{12}$ bringing together autophagosomal membranes and mitochondria via their LIR motif. ${ }^{4}$ In this regard, it is interesting to note that the absence of NIX results in mitochondrial retention and anemia in mice. ${ }^{12-14}$

The PINK1/Parkin pathway is one of the best-characterized pathways of mitophagy. In healthy functional mitochondria, PTEN-induced putative kinase 1 (PINK1) is addressed to the OMM. Once translocated to the inner membrane, this protein is cleaved and targeted for degradation by the proteasome. However, on defective mitochondria, there is an accumulation of PINK1 which results in the translocation of Parkin, an E3 ubiquitin ligase, and the subsequent clearance of damaged mitochondria by mitophagy. ${ }^{15-17}$

At the surface of damaged mitochondria, Parkin interacts with OMM proteins among which voltage-dependent anion channels (VDAC). Furthermore, in the absence of all three VDAC, mitophagy has been shown to be impaired, at least in certain cell types. ${ }^{18-20}$

VDAC1 is a ubiquitous OMM protein involved in different pathways including apoptosis, ${ }^{21,22}$ mitochondrial cristae scaffold, ${ }^{23}$ and nucleotide transport..$^{24,25}$ Its role in erythropoiesis is unknown, but transcriptomic and proteomic data could suggest a role at the terminal differentiation. ${ }^{26,27}$ Using a short hairpin RNA (shRNA) approach, we find that downregulation of VDAC1 results in a blockage in erythroid progenitor differentiation at the orthochromatic erythroblast stage, exhibiting a significantly decreased level of enucleation and cell death.

Furthermore, we show that the clearance of mitochondria from terminal erythroblasts is dependent on VDAC1, starting at the polychromatic erythroblast stage of differentiation. VDAC1 plays a crucial role to initiate the recruitment of phagophore's membrane, necessary to achieve an efficient maturation of human red blood cells.

\section{Methods}

\section{CD34 ${ }^{+}$cells isolation and ex vivo erythroid differentiation}

$\mathrm{CD} 4^{+}$cells were isolated from cord blood and cultured following a human ex vivo differentiation protocol as previously described $^{28}$ (see the Online Supplementary Appendix). The experimental protocol was approved by ethical committees from the Inspire H2020 program (agreement 655850) and from INTS
(2019-1), additional information is included in the Online Supplementary Appendix.

\section{Transduction of CD34 progenitors}

Cells undergoing erythroid differentiation were transduced at day 4 of erythroid differentiation with lentiviral vectors (MISSION ${ }^{\circledast}$ pLKO.1) containing scrambled shRNA (shSCR) or shRNA targeting VDAC1 (shVDAC1) (TRCN0000297481) upstream of the EGFP transgene, at a MOI of 10 (Online Supplementary Figure S1A). Transduction efficiency was evaluated by the percentage of $\mathrm{EGFP}^{+}$cells after 72 hours (h) and EGFP+ cells were sorted using the cell sorter SONY SH800.

\section{Flow cytometry-based analysis of erythroid differentiation}

Cells were analyzed for surface markers expression, mitochondrial content and the presence of a nucleus, starting at day 7. Briefly, $10^{5}$ cells were stained with $250 \mathrm{nM}$ MitoFluor and Hoechst 34580 in media for 30 minutes (min) at $37^{\circ} \mathrm{C}$. Cells were washed and subsequently stained with fluorochrome-conjugated antibodies against glycophorin A (GPA), Band3 and $\alpha 4$ integrin in phosphate-buffered saline (PBS) $2 \%$ bovine serum albumin (BSA), for $30 \mathrm{~min}$ at $4^{\circ} \mathrm{C}$. Cells were washed once with $\mathrm{PBS}$ and 7-AAD was added prior to acquisition to exclude dead cells. Cells were analyzed using a BD FACSCanto ${ }^{\mathrm{TM}}$ (BD Biosciences), data were acquired with Diva software and analyzed using FCS express 6 Flow Research Edition software.

\section{Enucleation assay by imaging flow cytometry}

Orthochromatic erythroblasts were sorted at day 12 as previously described.$^{28}$ Cells were maintained in culture for 1 day in phase III medium before staining with $1 \mu \mathrm{g} / \mathrm{mL}$ Hoechst 34580 and anti-GPA antibody. Nucleus polarization was analyzed by Amnis ${ }^{\circledR}$ Imaging Flow Cytometer. Approximately 10,000 events were collected and the "delta centroid GPA-Hoechst" was calculated as the distance of the center of the GPA-labeled erythroblast from the center of the Hoechst-labeled nucleus. A threshold of 2 for the delta centroid (DC) was fixed in order to discriminate between a polarized and non-polarized nucleus. Analysis was performed using the IDEAS 6.2 software. ${ }^{29}$

\section{Other standard methods}

Quantitative real-time polymerase chain reaction (qRT-PCR), antibodies and dyes, May-Grünwald-Giemsa (MGG)-based identification of erythroblasts stage, apoptosis assay, reactive oxygen species (ROS) detection, mitochondrial respiration analysis, immunoblotting, immunolabeling for confocal microscopy, ATP measurement, electron microscopy, K562 cell culture, image stream co-localization and statistical analysis are fully described in the Online Supplementary Appendix.

\section{Results}

\section{shRNA-mediated knockdown of VDAC1 results in an accelerated erythropoiesis until the orthochromatic erythroblast stage of differentiation}

In order to assess the specific effects of VDAC1 during erythroid differentiation, we pursued a shRNA-mediated knockdown approach. CD $34^{+}$cells were transduced at day 4 of differentiation with a shSCR or a shVDAC1 lentiviral vector, both harboring the EGFP transgene (Online Supplementary Figure S1A), and EGFP+ cells were sorted at day 7 (Figure 1A). Knockdown efficiency was assessed at day 10 and a significant downregulation of 
VDAC1 expression, both at the mRNA $(53.6 \pm 7.6 \%)$ and protein level $(78.5 \pm 5.9 \%)$ was observed as compared to shSCR-transduced cells (Online Supplementary Figure S1B and $C$ ). Of note, no significant difference was observed in the mRNA expression of two other VDAC, VDAC2 and
$V D A C 3$, confirming the specificity of the shRNA construct (Online Supplementary Figure S1D).

The effect of VDAC1 downregulation on erythroblast maturation was assessed by quantifying the percentages of erythroblasts at different stages of differentiation, as

A

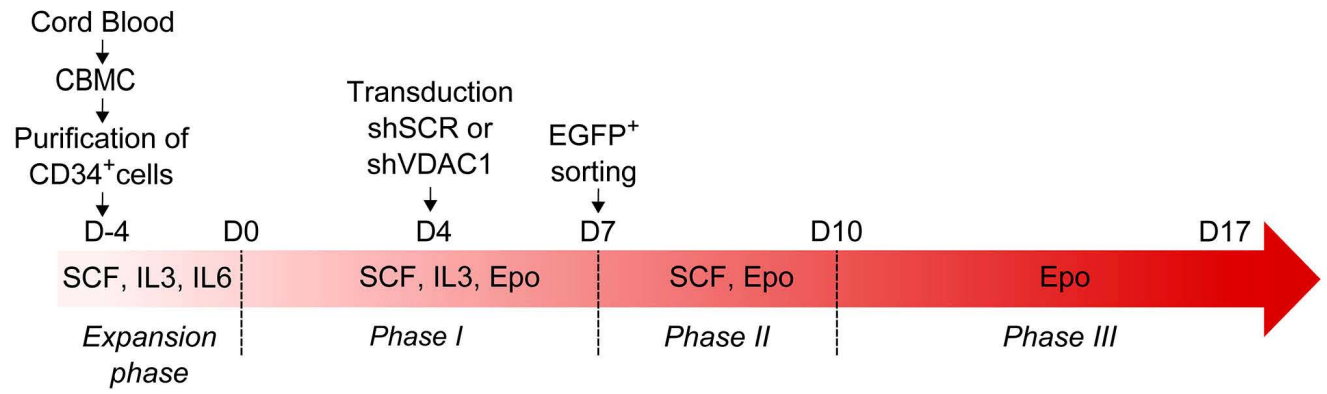

B
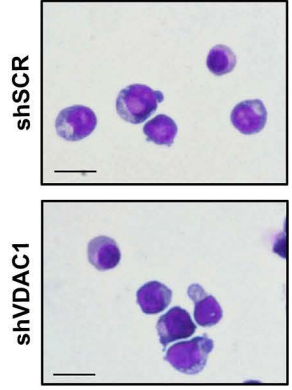

C
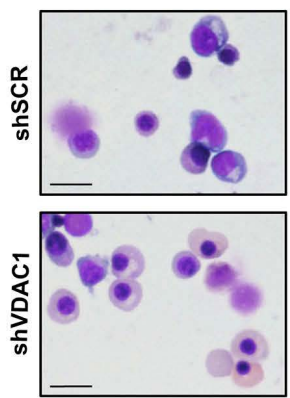

E

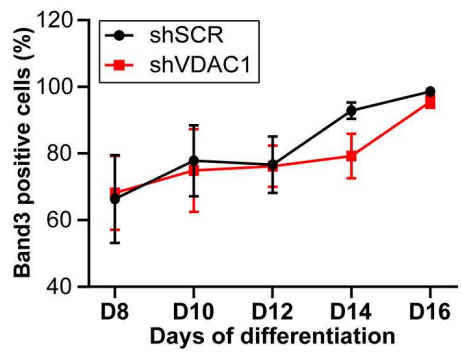

\section{D7}
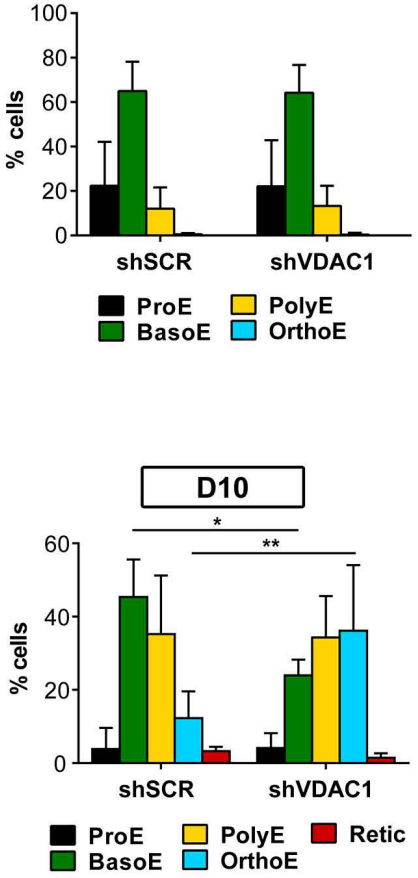

$\mathrm{F}$

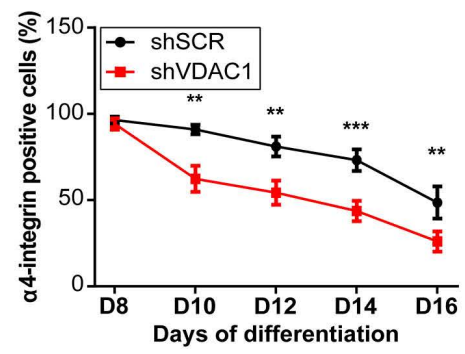

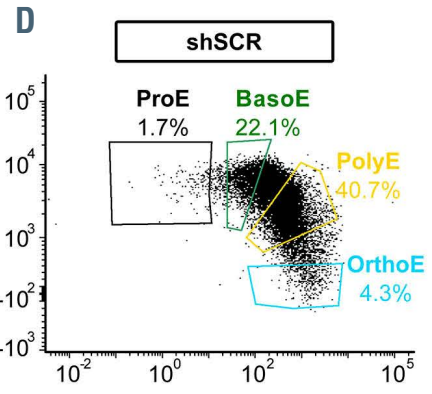

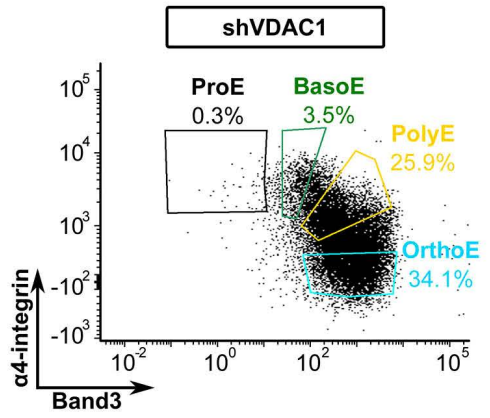

G

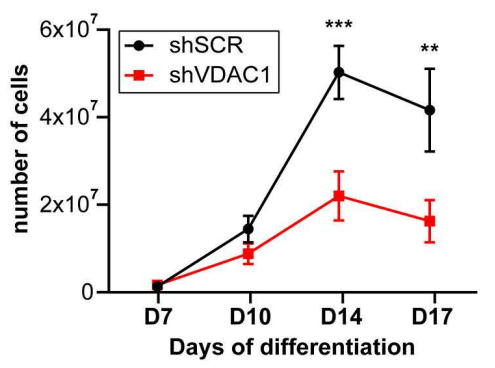

Figure 1. VDAC1 knockdown in human erythroid progenitors accelerates erythroid differentiation. (A) Schematic of the ex vivo erythroid differentiation protocol following short hairpin RNA (shRNA)-mediated downregulation of VDAC1. CD34+ progenitors were isolated from cord blood and transduced at day 4 with a lentiviral vector harboring either the VDAC1 shRNA or scramble shRNA (shSCR), together with the EGFP transgene. EGFP ${ }^{+}$cells were sorted at day 7 and differentiated until day 17. (B) Representative May-Grünwald-Giemsa (MGG) images of erythroid progenitors and quantification of cells at different stages of differentiation are shown for day 7 and $(C)$ day 10 of differentiation $(n=3)$. Scale bar $=20 \mu \mathrm{m}$. (D) Representative $\alpha 4$-integrin/Band3 flow cytometry profiles of GPA ${ }^{+}$cells at day 10 are shown, allowing different stages of differentiation to be distinguished. (E) Band3 and (F) $\alpha 4$-integrin surface expressions were evaluated on glycophorin A positive (GPA') cells following introduction of shSCR (black) and shVDAC1 (red) at days 8, 10, 14, and 16 of erythroid differentiation. The percentages at each time point were quantified and means \pm standard error are shown $(n=9)$. (G) Erythroblast proliferation in control progenitors and shVDAC1-transduced progenitors was monitored at days 7, 10, 14 and $17(\mathrm{n}=4) . * P<0.05, * * P<0.01, * * * P<0.001$. CBMC: cord blood mononucleated cells; shVDAC1: VDAC1 shRNA; D: day. ProE: proerythroblast; BasoE: basophilic erythroblasts; PolyE: polychromatic erythroblasts; OrthoE: orthochromatic erythroblasts; Retic: reticulocytes. 
monitored by MGG coloration. VDAC1 downregulation did not alter erythroid differentiation before 3 days posttransduction (D7) (Figure 1B). However, by day 10 of differentiation, shVDAC1 transduced cells exhibited an accelerated terminal differentiation as compared to control cells, shown by a significantly higher percentage of orthochromatic erythroblasts as compared to earlier basophilic and polychromatic erythroblasts (Figure 1C). These data were confirmed by evaluating $\alpha 4$ integrin/Band3 profile by flow cytometry allowing the terminal stage of differentiation to be distinguished as previously described ${ }^{28}$ (Figure 1D). Consistent with the data above, shVDAC1-transduced progenitors showed no difference in Band3 expression (Figure 1E) but a significantly decreased levels of $\alpha 4$ integrin, a marker which is lost during terminal differentiation, as compared to shSCR-transduced cells (Figure 1F). Furthermore, we observed a decreased proliferation rate in shVDAC1transduced cells starting at day 10 (Figure 1G). Altogether, these data point to the importance of VDAC1 in regulating terminal erythroid differentiation.

\section{VDAC1 downregulation impairs erythroblast enucleation}

The main morphological feature of terminal mammalian erythroid differentiation is the enucleation, leading to the production of a reticulocyte and a pyrenocyte from an orthochromatic erythroblast. Despite the accelerated differentiation observed in shVDAC1-transduced cells, orthochromatic erythroblast percentage was increased whereas reticulocyte level was significantly decreased from day 14 until day 17 as evaluated by MGG coloration (Figure 2A and B) as well as Hoechst staining (Figure 2C).

Enucleation starts with the polarization of the nucleus towards the plasma membrane, allowing the separation of the nucleus from the yet-to-be formed reticulocyte by a mechanism similar to cytokinesis. ${ }^{30}$ In order to assess whether the decrease in enucleation in shVDAC1-transduced cells was due to an impairment in the polarization of the nucleus, we sorted orthochromatic erythroblasts at day 12 of differentiation and analyzed these cells by imaging flow cytometry 24 hours (h) later. We measured the distance between the center of the cell and the center of the nucleus (delta centroid XY from the IDEAS software). No significant differences in delta centroid XY were observed between shVDAC1- and shSCR-transduced cells, suggesting that VDAC1 downregulation does not block the polarization of the nucleus (Online Supplementary Figure S2).

In order to assess whether the block in enucleation affects cell viability, we evaluated the level of apoptosis as a function of annexin $V$ staining of exposed phosphatidylserine. While annexin $\mathrm{V}$ staining was not altered in shVDAC1-transduced cells as compared to shSCRtransduced cells at days 7 and 10 of differentiation, levels were significantly higher at days 14 and 17, strongly suggesting that an attenuated enucleation was associated with apoptosis (Figure 2D).
A
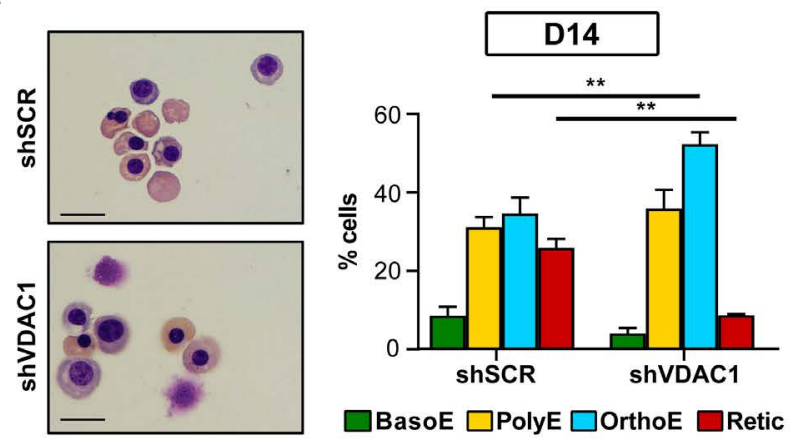

B

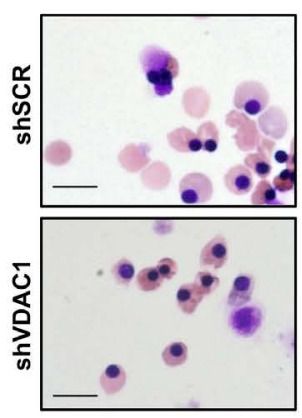

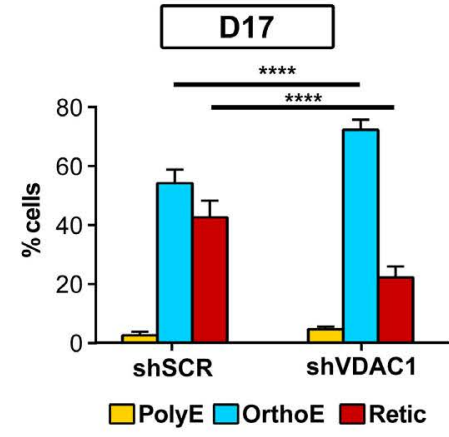

C

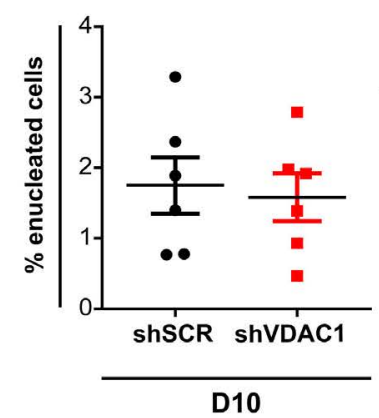

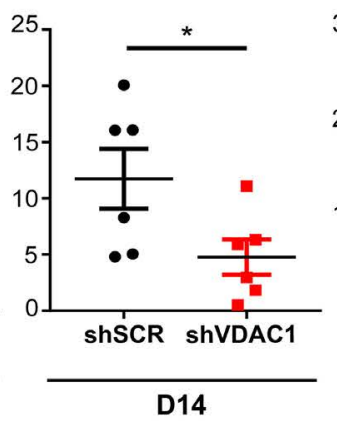

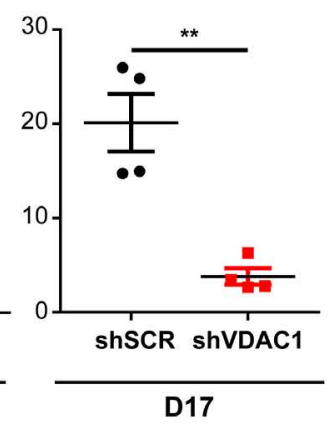

D

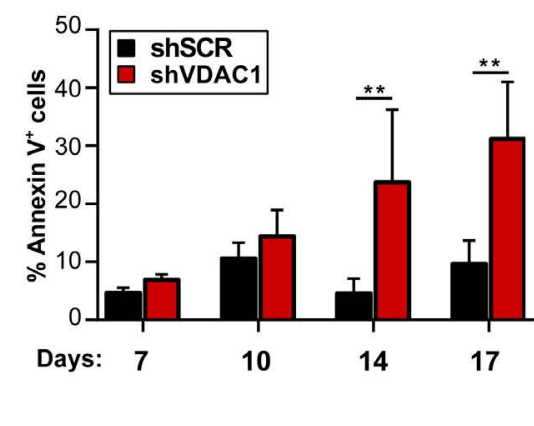

Figure 2. Enucleation is impaired in short hairpin VDAC1-transduced erythroid progenitors. (A) The progression of terminal erythroid progenitors to the reticulocyte stage of differentiation was evaluated following scramble shRNA (shSCR) and VDAC1 shRNA (shVDAC1) transduction. Representative May-Grünwald-Giemsa (MGG) images (left) and quantification of the different progenitor stages (right) at day 14 and (A) day 17 (B) are presented and means \pm standard error (SE) are shown. Scale bar $=20 \mu \mathrm{m}$. (C) The percentages of enucleated cells (glycophorin A postive [GPA] , Hoechst cells) were quantified by flow cytometry at day 10,14 and 17 of differentiation $(n=4)$ and means $\pm S E$ are shown. (D) Apoptosis was evaluated by annexin $V$ staining and the quantification of annexin $V^{+}$cells is presented at day 7 , 10, 14 and $17(\mathrm{n}=3) . * P<0.05, * * P<0.01, * * * * P<0.0001$. BasoE: basophilic erythroblasts; PolyE: polychromatic erythroblasts; OrthoE: orthochromatic erythroblasts; Retic: reticulocytes. 
Mitochondrial biomass remains elevated in shVDAC1-transduced erythroblasts

In mammals, mature red blood cells are devoid of mitochondria. However, mitochondria can still be found at the reticulocyte stage in many mammalian species such as rabbits, dogs, rats, mice and humans..$^{5,31-33}$ As expected, we detected a progressive decrease in mitochondrial biomass during erythroblast maturation, with a first major decrease in the transition between basophilic and polychromatic stages and a second between polychromatic and orthochromatic stages (Figure 3A). At day 10 of differentiation the mitochondrial marker TOM40 was detected at significantly higher levels in shVDAC1-transduced cells by western blot analysis (2.6 \pm 0.6 -fold, $n=5$; Figure $3 \mathrm{~B}$ ). As VDAC1 downregulation alters the kinetics of differentiation, difference on protein expression at day 10 could results from a different proportion of erythroblastic stages. For this reason, mitochondrial biomass was assessed by flow cytometry in the different stages based on $\alpha$-4integrin/Band3 profile as previously described. Mitochondrial biomass was significantly higher in shVDAC1-polychromatic and orthochromatic erythroblasts as compared to shSCR-transduced erythroblasts (Figure 3C). Importantly, these differences were associated with late stages of differentiation as there was no change in mitochondrial biomass in earlier basophilic erythroblasts. Although shSCR transduced cells progressively eliminate mitochondria at each differentiation stage, our data point to a defect in mitochondrial clearance dur- ing the transition between basophilic and polychromatic erythroblasts in conditions of VDAC1 downregulation.

\section{VDAC1 regulates mitochondrial morphology and oxidative phosphorylation in terminal erythroblasts}

In order to evaluate mitochondrial function in shVDAC1-transduced erythroblasts, we first evaluated the structural morphology of mitochondria by transmission electron microscopy at day 10 of differentiation (Figures 4A; Online Supplementary Figure S3). In contrast with the normal ultrastructural morphology detected in shSCR-transduced cells, shVDAC1-transduced cells exhibited swollen mitochondrial cristae (Figure 4A and B) with a significantly increased percentage of rounded as compared to elongated mitochondria $(P<0.0001$, Figure $4 \mathrm{~A}$ to $\mathrm{C})$. These data are in agreement with a published role of VDAC1 OMM-IMM contacts sites in cristae structuration. ${ }^{23}$ Interestingly, we also observed a reduced number of ER-mitochondria contact sites in shVDAC1-transduced cells (Figure 4D). As these mitochondrial associated membranes have been shown to recruit signaling molecules such as mTOR, GSK3 and hexokinase I, thereby increasing oxidative phosphorylation, ${ }^{34}$ it was of interest to evaluate mitochondrial function in erythroblasts with downregulated VDAC1. Notably, shVDAC1-transduced erythroblasts exhibited a significantly decreased oxygen consumption rate, evaluated as a function of mitochondrial biomass (Figure 4E). This measure, quantifying oxidative phosphorylation
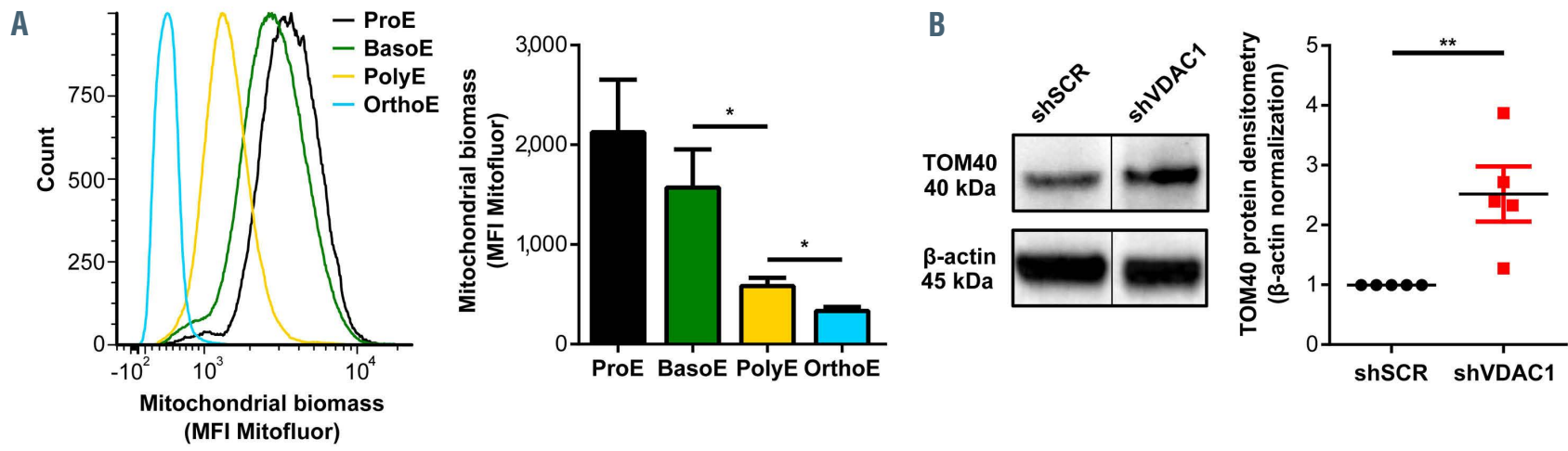

C
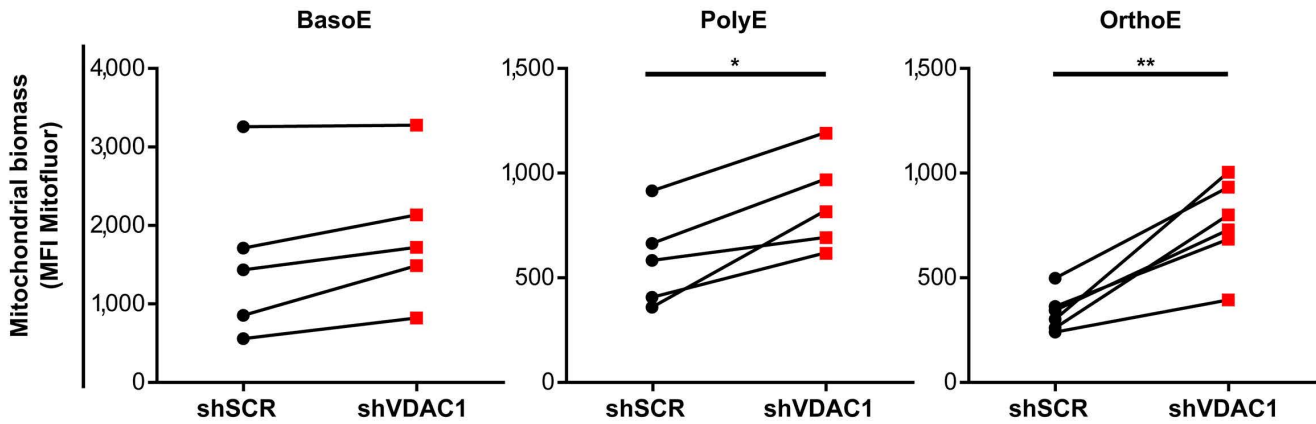

Figure 3. VDAC1 downregulation causes a retention of mitochondria in late stage erythroblasts. (A) Mitochondrial mass was measured by Mitofluor staining in erythroblasts at different stages of terminal differentiation and representative histograms (left) and quantification of the mean fluorescence intensity (MFI) are shown $(n=5)$ (right). (B) Levels of the mitochondrial protein TOM40 were quantified by immunoblot at day 10 of differentiation and representative blots (left) as well as normalization to $\beta$-actin (right) are presented. Levels in control cells was arbitrarily set at "1" $(n=5)$. (C) Mitochondrial content of erythroblasts at day 11 of differentiation was evaluated by Mitofluor staining on basophilic (BasoE), polychromatic (PolyE) and orthochromatic (OrthoE) erythroblasts and MFI in progenitors transduced with scramble shRNA (shSCR) (black dots) and VDAC1 shRNA (shVDAC1) (red dots) transduced cells are presented ( $n=5)$. Gates for erythroblasts populations are based on $\alpha 4$-integrin/Band3 profiles as shown in Figure 1D. $* P<0.05, * * P<0.01$. ProE: proerythroblast. 
(OXPHOS), was lower in shVDAC1-transduced erythroblasts for both basal levels and maximal respiration (Figure 4F). In accord with the lower OXPHOS, intracellular ATP levels were significantly lower in shVDAC1transduced cells than control cells (Figure 4G). We also measured mitochondria membrane potential and ROS accumulation at day 10 and showed no difference in cells transduced with shVDAC1 compared to the shSCR (Online Supplementary Figure S4), suggesting an adaptation of the cell to compensate for mitochondria changes of metabolism upon VDAC1 downregulation. Thus, decreased expression of VDAC1 attenuates mitochondr- ial function, resulting in a diminished energetic state of the differentiating erythroblast.

\section{Delayed mitochondrial clearance in late-stage ery- throblasts with downregulated VDAC1 is due to a} defective recruitment of autophagosomal membranes

Given that VDAC1 has been shown to regulate mitophagy in non-erythroid cell lines ${ }^{18-20}$ we hypothesized that VDAC1 downregulation could impair mitophagy in human erythroblasts as well. While the autophagy-mediated lipidation of LC3-I to LC3-II in the autophagosome membrane was not altered upon VDAC1 downregulation
A
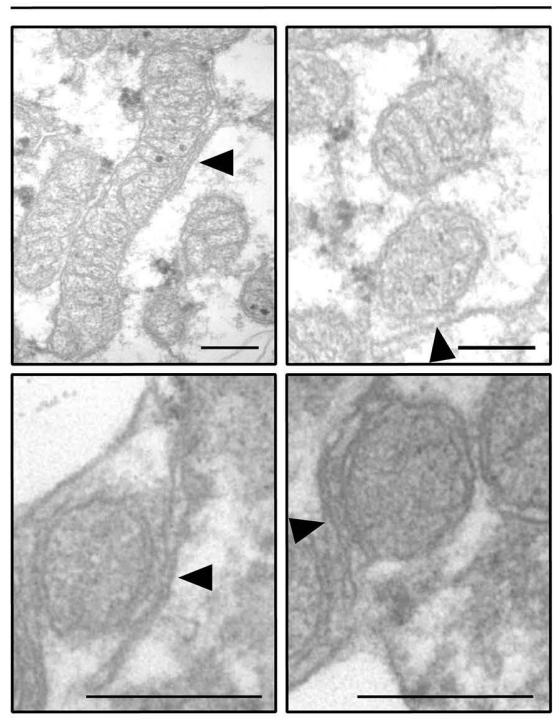

D

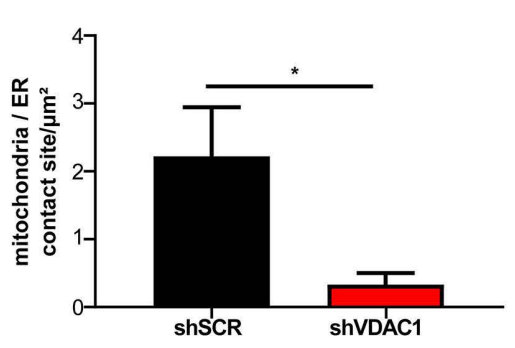

D10 shVDAC1
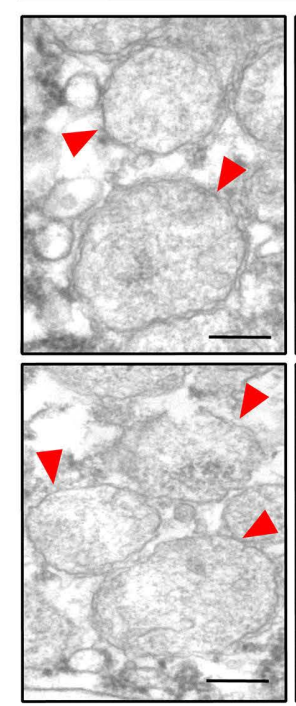
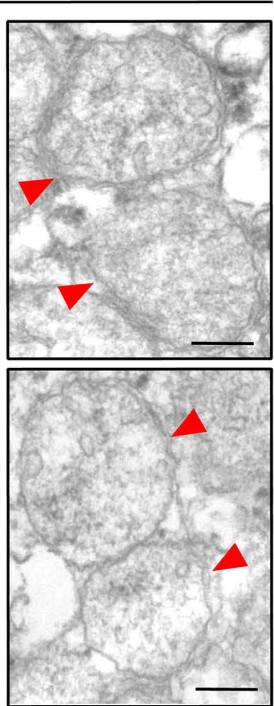

B

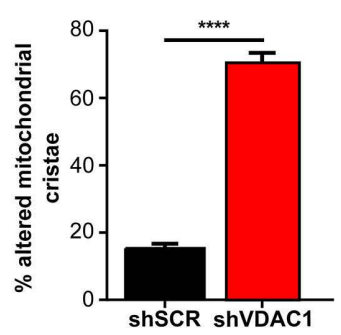

C

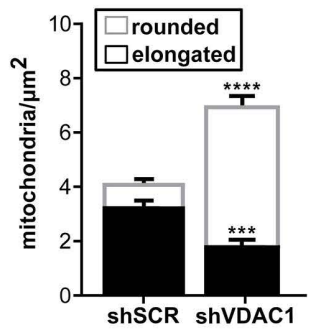

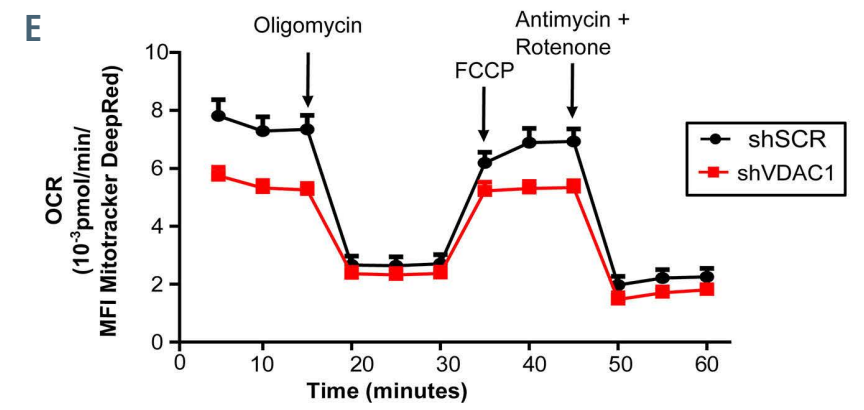

$\mathrm{F}$
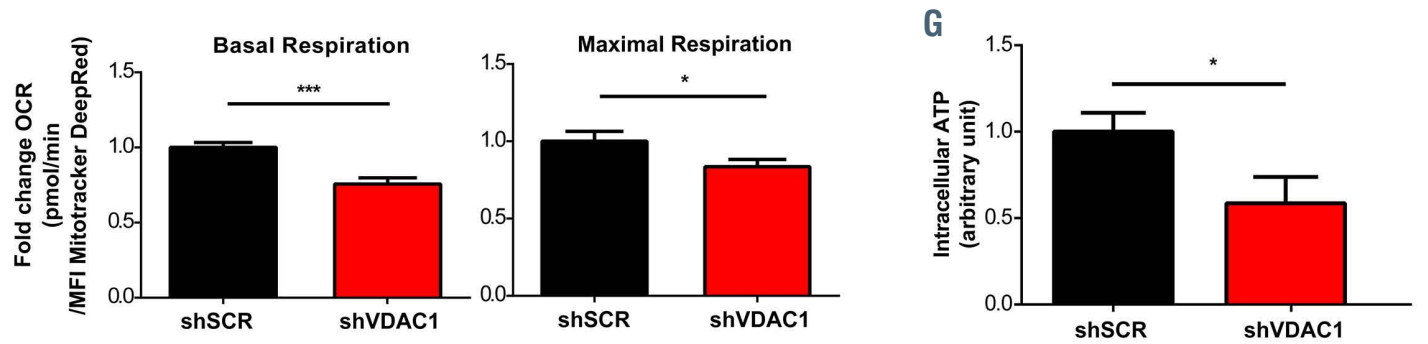

Figure 4. Mitochondria morphology is altered and oxidative phosphorylation is attenuated upon VDAC1 downregulation. (A) Sample transmission electron microscopy (TEM) images of erythroblasts at day 10 (D10) of differentiation (black arrows show endoplasmic reticulum [ER]-mitochondria contact site) with swollen cristae indicated by red arrows. Scale bar $=200 \mathrm{~nm}$. (B) TEM-based quantification of the percentage of altered cristae and (C) number of rounded (white) and elongated (black) mitochondria by $u^{2}{ }^{2}$ are presented. (D) The number of mitochondria-ER contact site by $\mathrm{m}^{2}$ is presented. Quantification in 20 cells for panels $B$ and $C$ and nine different cells for panel D. $* P<0.05, * * * P<0.001, * * * * P<0.0001$. (E) Oxygen consumption rates (OCR) were monitored in the indicated populations at $\mathrm{D} 10$ of differentiation by Seahorse technology and representative profiles are formed ( $\mathrm{n}>3$ technical replicates). Data were normalized to mitochondrial quantity using mean fluorescence intensity (MFI) values of mitotracker deep red previously measured by flow cytometry. (F) Basal and maximum respiration were quantified with levels in control cells arbitrarily set at “1” $(n=3)$. (G) Intracellular ATP in scramble shRNA (shSCR) and VDAC1 shRNA (shVDAC1)-transduced erythroblasts was evaluated at D10 of differentiation by luminometry with levels in control cells arbitrarily set at " 1 " $(n=3)$. Data are presented as means \pm standard error. * $P<0.05$, $* * * P<0.001$ 
A

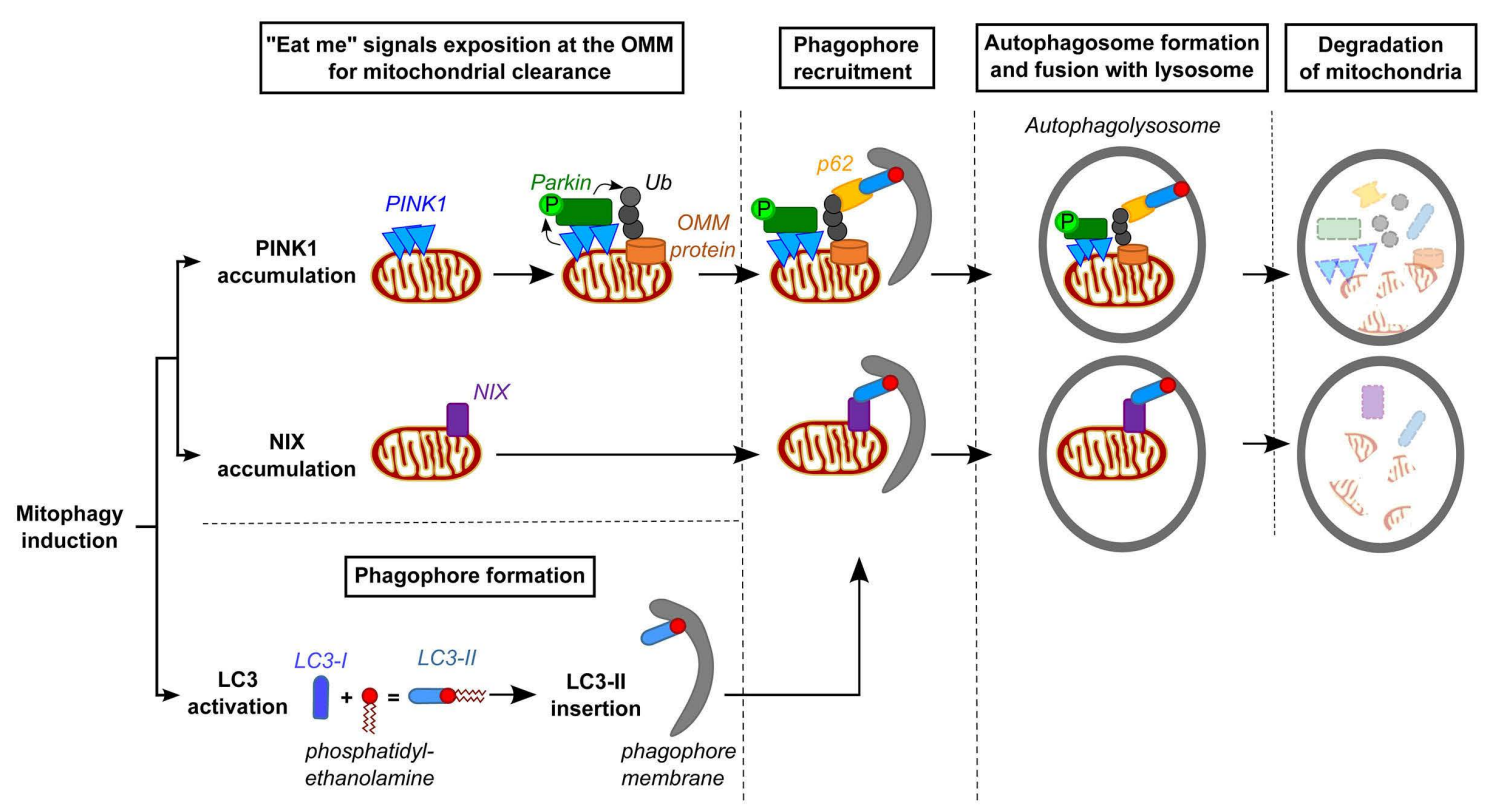

B

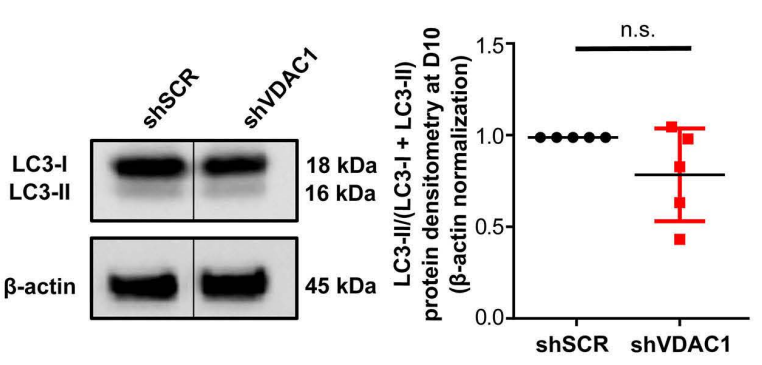

C

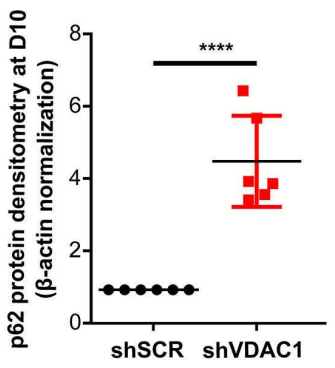

D
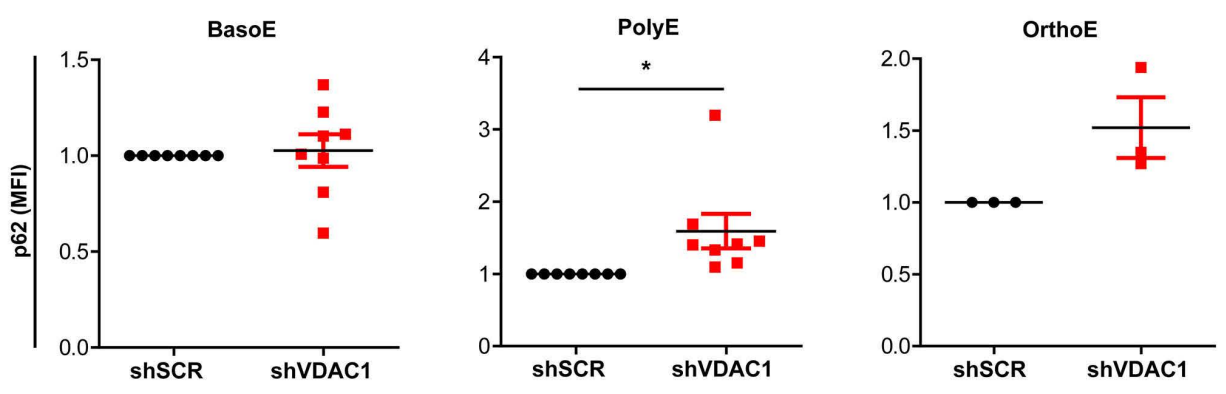

E

LC3

Tом22
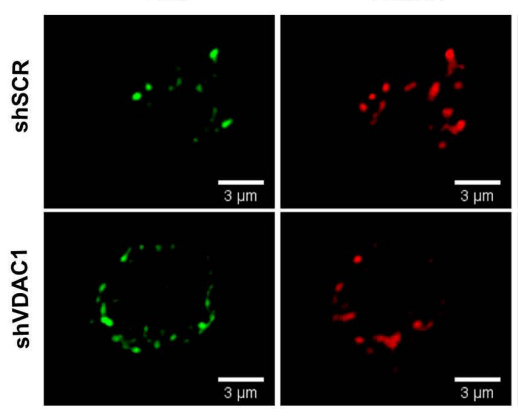

Merge
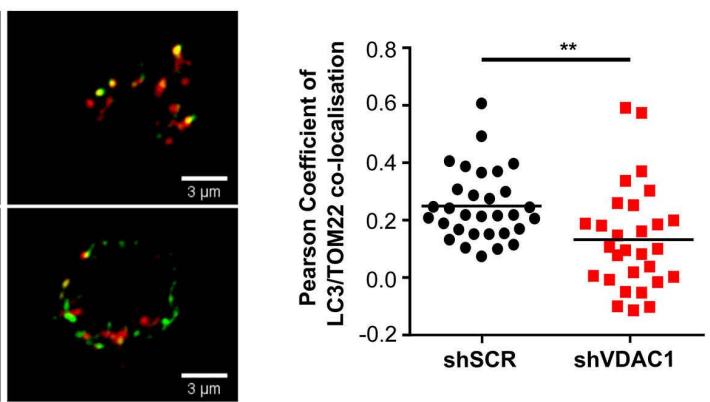

Figure 5. Autophagy is impaired in VDAC1 scramble short hairpin RNA-transduced cells. (A) Schematic representation of the role of the PINK1/Parkin and NIX pathways in mitochondrial degradation. (B) LC3-I and LC3-II levels were evaluated in scramble shRNA (shSCR) and VDAC1 shRNA (shVDAC1)-transduced erythroblasts at day 10 (D10) and representative immunoblots are presented (left). LC3-II levels were normalized to $\beta$-actin with levels in control cells arbitrarily set at "1" ( $n=5)$ (right). (C) p62 levels were monitored and representative immunoblots (left) and quantifications (right) are presented. p62 levels were normalized to $\beta$-actin with levels in control cells arbitrarily set at " 1 " ( $n=6)$. (D) p62 protein level was evaluated by flow cytometry on basophilic (BasoE), polychromatic (PolyE) ( $n=8)$ and orthochromatic (OrthoE) $(n=3)$ and mean fluorescence intensity (MFI) of shSCR (black dots) and shVDAC1 (red dots) are presented with levels in control cells arbitrarily set at "1". Gates for erythroblasts populations are based on $\alpha 4$-integrin/Band3 profiles as shown in Figure 1D. * $P<0.05$. (E) Co-localization of LC3 and TOM22 was monitored by confocal microscopy and representative images are shown. Scale bar $=3 \mu \mathrm{m}$. (left) The Pearson coefficient between LC3 and TOM22 are presented for 30 cells in a representative experiment $(\mathrm{n}=3)$ (right). ${ }^{*} P<0.05, * * P<0.01, * * * * P<0.0001$. ns: non-significant. 
(Figure 5B), there was a significant accumulation of $\mathrm{p} 62$, an adaptor protein that allows bridging between the ubiquitinated cargos and the phagophore membrane ${ }^{35}$ (Figure $5 \mathrm{~A}$ to $\mathrm{C}$ ). Furthermore, p62 expression was detected by flow cytometry in different stages on fixed cells stained with $\alpha 4$-integrin and Band3 antibodies. As well as for mitochondrial content, a significant increase in p62 was observed at polychromatic and orthochromatic stages in shVDAC1-transduced cells (Figure 5D).

After engulfment of the mitochondria, the autophagosome usually fuses with a lysosome, leading to the degradation of the mitochondria along with the adaptor proteins (Figure 5A). In order to decipher whether p62 accumulation was due to a stabilization at MOM on ubiquitinated proteins or to a defect in mitophagy completion by lysosomal degradation, we studied the autophagosomal membrane recruitment to mitochondria by immunofluorescence. We observed a significant decreased co-localization between autophagosomal LC3-II and the mitochondrial marker TOM22 in shVDAC1-transduced cells $(P<0.01$; Figure $5 \mathrm{E})$, suggesting a defect in the phagophore membrane recruitment and consequently a defect in mitophagy completion.

In order to assess the specificity of our results, we constructed shSCR-K562 and shVDAC1-K562 stable cell lines and transfected shSCR-K562 cells with a small interfering RNA (siRNA) against VDAC1 (siVDAC1-K562). We confirmed VDAC1 downregulation by western blot (Online Supplementary Figure S5A), and showed, as for differentiating erythroblasts, no difference in LC3 activation (Online Supplementary Figure S5B) and an increase in p62 protein level (Online Supplementary Figure S5C). We also observed a decrease of LC3/TOM22 co-localization in shVDAC1-K562 and siVDAC1-K562, which indicates that the recruitment of the phagophore membrane to the mitochondria was impaired upon VDAC1 downregulation (Online Supplementary Figure S5D). These data confirmed that VDAC1 downregulation in K562 erythroid cells recapitulates the phenotype observed on VDAC1downregulated erythroblasts.

\section{PINK1 protein level is reduced upon VDAC1 downregulation}

The phagophore recruitment is dependent on the stabilization of some specific MOM proteins and direct or indirect interaction with lipidated LC3 (Figure 5A). We first evaluated NIX levels, previously identified in mouse reticulocytes as being critical for the recruitment of autophagosomal membranes and appropriate mitophagy. ${ }^{36}$ Notably, we detected no significant decrease in neither NIX dimers nor NIX transcripts in human erythroblasts with decreased VDAC1 expression (Online Supplementary Figure S6). We then studied the second main mitophagy induction pathway and observed a significant decrease of full-length PINK1 protein level in shVDAC1-transduced bulk erythroblasts at D10 by western blotting (Figure 6A). PINK1 expression was then evaluated by flow cytometry on basophilic, polychromatic and orthochromatic erythroblasts based on the $\alpha 4$-integrin and Band3 expression pattern. We confirmed a significant decrease of PINK1 level starting from the polychromatic stage (Figure $6 \mathrm{~B}$ ), in line with the differences of mitochondrial mass and p62 expression.

We next confirmed the reduction of PINK1 protein level upon VDAC1 downregulation in K562 cell lines (Figure
6C). Furthermore, we observed a reduction of the mitochondrial localization of PINK1, as shown by the reduction of PINK1/TOM22 co-localization in shVDAC1-K562 cells (Figure 6D). Finally, we observed a decrease of the phagophore recruitment to the mitochondria when downregulating PINK1 in shSCR-K562, as observed with the significant reduction of LC3/TOM22 co-localization (Figure 6E). In contrast, LC3/TOM22 co-localization did not worsen upon PINK1 downregulation in shVDAC1K562 cells (Figure 6E).

\section{Discussion}

Mitochondrial clearance is a crucial process for the production of mature red blood cells and several studies have shown that mitophagy occurs at the reticulocyte stage, allowing the complete clearance of mitochondria and leading to final murine erythrocyte maturation. ${ }^{13,14}$ Mitophagy is a mitochondrial-specific form of general macroautophagy, which can be mediated by different well characterized pathways involving protein signaling as the PINK1/Parkin pathway, NIX and BNIP3. While autophagy related genes (ATG) have been shown to be critical for mitochondrial clearance during erythropoiesis, ${ }^{6-11}$ and the NIX-driven pathway has been identified as plying a role in murine erythropoiesis, ${ }^{12-14}$ the importance of these mitophagy pathways in human erythroid terminal differentiation is unknown.

Here we focused on the role of VDAC1, an OMM protein, known to be involved in mitophagy in non-erythroid cells.

We found that VDAC1 downregulation accelerates human erythroblast differentiation until the orthochromatic erythroblast stage, but then blocks enucleation and cumulates mitochondria with altered ultrastructure and decreased function, resulting in apoptosis.

In agreement with previous data on the role of VDAC1 in the structuration of mitochondria, ${ }^{23}$ we observed altered mitochondria ultrastructure and a decrease in ERmitochondria contact sites in shVDAC1-transduced erythroblasts by electron microscopy. Notably, complexes between VDAC and hexokinase-1 at ER-mitochondrial contact sites have been shown to regulate mitochondrial metabolism. ${ }^{34}$ In our study, mitochondria in VDAC1downregulated erythroblasts showed a decrease in OCR and intracellular ATP content, a signature of reduced mitochondrial activity. Thus, perturbations in mitochondrial metabolism may influence the kinetics of erythroblast differentiation and their enucleation profiles.

We demonstrated that VDAC1 is required for progressive mitochondrial clearance during the terminal phase of differentiation. This is in line with a previous study showing a decrease in mitochondrial mass after the proerythroblastic stage. ${ }^{37} \mathrm{We}$ found that this is regulated through the PINK1/Parkin pathway, with VDAC1 modulating PINK1 accumulation at the mitochondria outer membrane and the recruitment of the phagophore. Accordingly, the attenuation of mitochondrial clearance was associated with increased levels of p62, an adaptor protein that allows bridging between the ubiquitinated cargos and the phagophore membrane..$^{35}$

Our data therefore highlight the critical role of VDAC1 function in the recognition of mitochondria that are "to be degraded" during the process of red cell maturation. 
We recently demonstrated that TSPO1 downregulation also affects mitochondria clearance with no effect on the erythroblasts' differentiation kinetics, ${ }^{38}$ raising the possibility that this phenotype is mediated through the VDAC1-TSPO1 OMM complex.
In addition, our findings suggest that the canonical PINK1/Parkin pathway may play an important role during human erythropoiesis, confirming in humans a previous study on mice. ${ }^{39}$ Transcriptome analyses in human erythroblasts detected an upregulation, starting from the
A
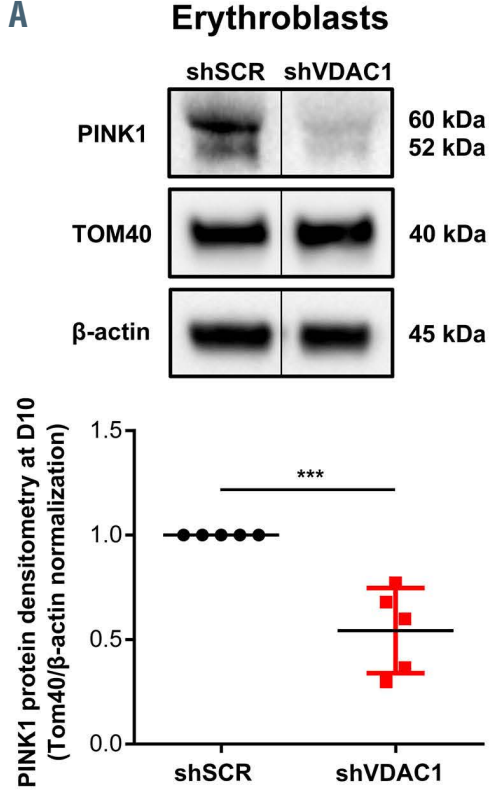

C
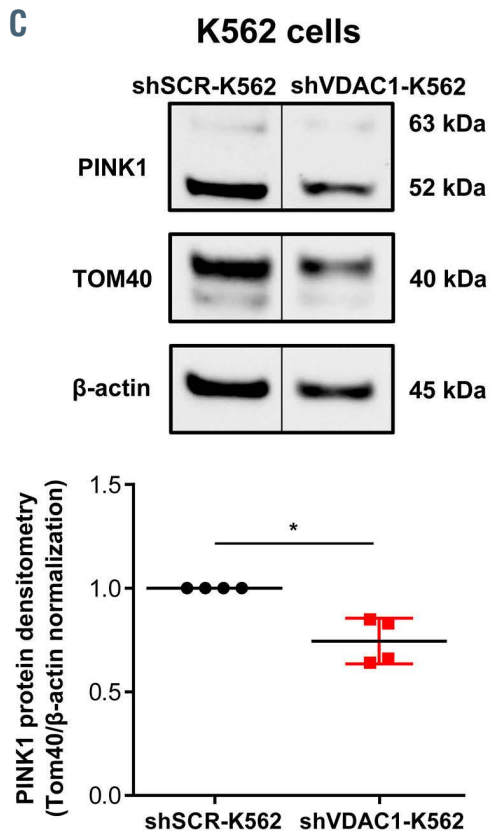

B

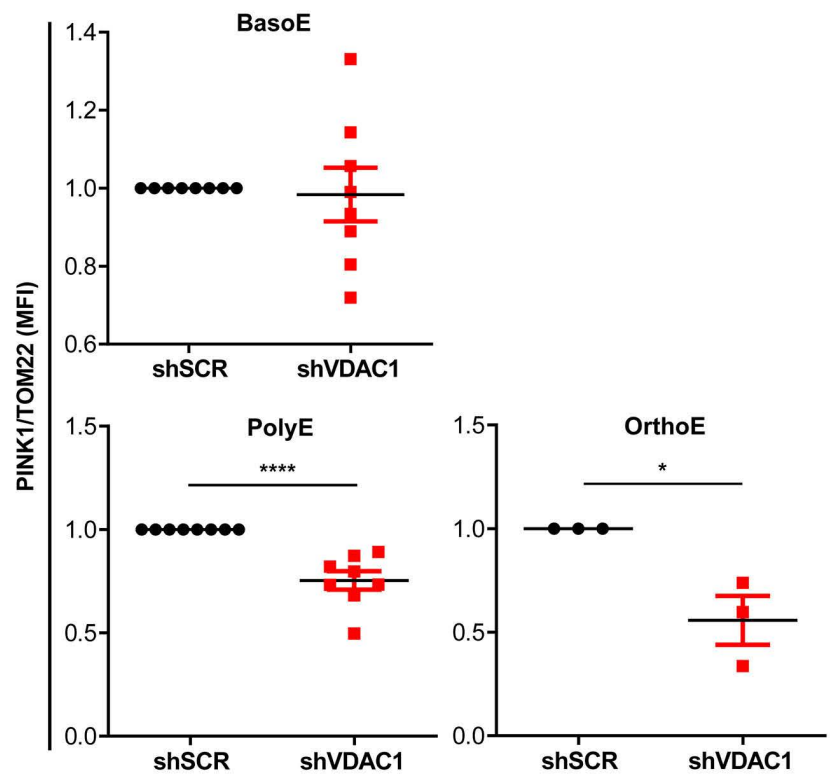

D

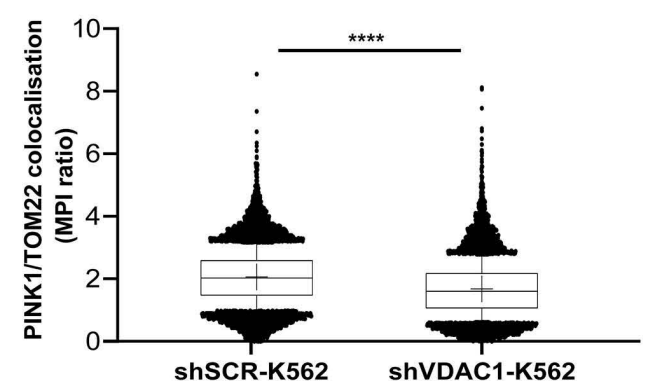

$\mathrm{E}$

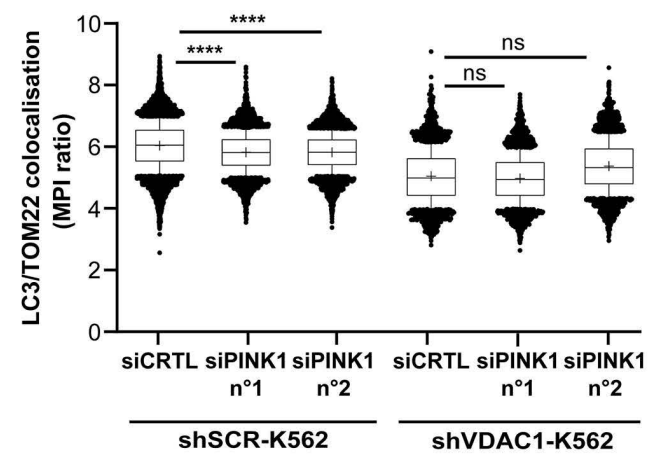

Figure 6. Lower PINK1 protein levels upon VDAC1 downregulation. (A) PINK1 levels were evaluated by immunoblot in the indicated conditions at day 10 of differentiation and normalized to the quantity of mitochondria (housekeeping mitochondrial TOM40) on total number of cell (housekeeping $\beta$-actin) (top). Levels in control cells were arbitrarily set at "1" $(n=5)$ (bottom). (B) PINK1 protein level was evaluated by flow cytometry on basophilic (BasoE), polychromatic (PolyE) ( $n=8)$ and orthochromatic (OrthoE) erythroblasts ( $n=3$ ). PINK1 mean fluorescence intensity (MFI) was normalized to mitochondrial housekeeping TOM22 MFI in erythroblasts transduced with scramble shRNA (shSCR) (black dots) and VDAC1 shRNA (shVDAC1) (red dots), with levels in control cells arbitrarily set at "1". Gates for erythroblasts populations are based on $\alpha 4$-integrin/Band3 profiles as shown in Figure 1D. (C) PINK1 protein levels were evaluated by immunoblot in the indicated conditions in shSCR-K562 and shVDAC1-K562 cells and normalized to the quantity of mitochondria marker TOM40 on total number of cell ( $\beta$-actin). Levels in control cells were arbitrarily set at "1" (n=4). (D) PINK1/TOM22 co-localization (MPI ratio from PINK1 and TOM22 signals) assessed by imaging flow cytometry of shSCR-K562 and shVDAC1-K562 cells $(n=3)$. (E) LC3/TOM22 co-localization assessed by imaging flow cytometry (myeloma prognostic index [MPI] ratio from LC3 and TOM22 signals) of shSCR-K562 and shVDAC1-K562 cells and shSCR-K562 cells transfected with a PINK1 siRNA $(n=3) . * P<0.05, * * \star P<0.001$, $* \star \star \star P<0.0001$. ns: non-significant; OMM: outer mitochondrial membrane. 
polychromatic stage, of key proteins of mitophagy belonging to different pathways, such as NIX and PINK1. ${ }^{26}$ NIX upregulation was described by several works studying NIX-mediated mitophagy in murine reticulocytes, ${ }^{12-14}$ but no clear evidence was found to explain the upregulation of PINK1. Under the condition of VDAC1 downregulation, NIX protein levels were not altered, suggesting that mitochondrial retention was not due to a defect in the NIX pathway but in the PINK1 pathway, as described below. It was previously reported that VDAC is required to promote Parkin recruitment to the mitochondria. ${ }^{18}$ Our data suggest that this could be due to a lack in PINK1 accumulation at the OMM.

Since VDAC1 is part of a multimeric complex with ATAD3A, ${ }^{23}$ we speculate that in the absence of VDAC1, ATAD3A is free to form alternative complexes. ${ }^{40}$ Recently, a new function of ATAD3A was described in hematopoietic progenitor cells, promoting the import of PINK1 ${ }^{41}$ In this context PINK1 is rapidly degraded by the mitochondrial peptidases MPP and Parl to prevent its accumulation in the surface prevent mitophagy. However, when ATAD3A dissociates from the translocase of the inner membrane (TIM) complex, the import of PINK1 is blocked, causing its accumulation. ${ }^{42}$ It is noteworthy that also hexokinase 2 (HK2) and adenine nucleotide transporter (ANT), both proteins known to be involved in a multimeric mitochondrial membrane protein complex, have been recently shown to be essential for PINK1 accumulation at the OMM, ${ }^{43,44}$ suggesting a role of this complex in PINK1/Parkin mitophagy. Further studies are necessary to assess this hypothesis and evaluate the crosstalk between VDAC1, ATAD3A, ANT and/or HK2 in the terminal phase of erythropoiesis.

In conclusion, our data are the first demonstration of a fundamental role of mitochondrial-selective autophagy at the erythroblast stage. Loss of VDAC1 causes an ineffective elimination of mitochondria in the transition between basophilic and orthochromatic erythroblasts, demonstrating the critical role of VDAC1 in late stage erythropoiesis.

We have taken account of recent reports highlighting the possible unspecific and strain-dependent effects of shRNA in knocking down mouse erythroblasts enucleation. ${ }^{45}$ However, we have observed the same effects after knocking down VDAC1 by two different approaches (shRNA and siRNA) and by using different experimental models, strongly supporting our conclusions.
Over the last few years, several studies have demonstrated the association of mitophagy defects with various hematological syndromes, highlighting the importance of mitochondrial content and mitophagy during human erythropoiesis. ${ }^{46-53}$ Our work provides a better understanding of the mechanisms regulating mitochondrial autophagy in human erythroid progenitors, particularly during the terminal phase of erythropoiesis. Elucidating the different pathways involved in mitochondrial clearance and their kinetics, will promote the development of novel therapeutic approaches for treating hematological disorders that involve defective erythroid maturation.

\section{Disclosures}

No conflicts of interest to disclose

\section{Contributions}

$M M$ designed experiments, performed experiments, analyzed data and wrote the paper; $C H, P G M, M D, S K$ and $C F$ performed experiments; JL evaluated protocols and supervised blood samples purchasing; CLVK critically evaluated experiments and obtained funding; SK and NT critically evaluated experiments, provided supervision, wrote the paper and obtained funding; SDL and MAO conceived the project, obtained funding, designed and critically evaluated experiments, provided supervision and wrote the paper; SDL and MAO contributed equally to this paper. All authors read and commented on the paper and approved the final version.

\section{Acknowledgments}

The authors would like to thank Mrs Corentine Chrysostome et Mr Abdellah Nait for their secretarial and technical assistance, respectively. We acknowledge the Cell Sorting facility of the Institut Imagine; the ImagoSeine core facility of the Institut Jacques Monod, member of IBiSA and France-BioImaging (ANR-10-INBS-04) infrastructure; the Laboratory of Excellence GR-Ex (Grant ANR-11-LABX-0051) and the Guests Researcher program from Paris Diderot University.

\section{Funding}

$M M$ is funded by the European Union's Horizon 2020 research and innovation program under the Marie SktodowskaCurie grant agreement No. 665850, the Club du Globule Rouge et du Fer (CGRF) and Société Française d'Hématologie (SFH); $P G-M$ is founded by the CLARIN-COFUND program from the Principado de Asturias and the European Union.

\section{References}

1. Palis J. Primitive and definitive erythropoiesis in mammals. Front Physiol. 2014:5:3.

2. Moras M, Lefevre SD, Ostuni MA. From erythroblasts to mature red blood cells: organelle clearance in mammals. Front Physiol. 2017;8:1076

3. Klionsky DJ. The molecular machinery of autophagy: unanswered questions. J Cell Sci. 2005;118(Pt 1):7-18.

4. Liu L, Sakakibara K, Chen O, Okamoto K. Receptor-mediated mitophagy in yeast and mammalian systems. Cell Res. 2014;24(7):787-795.

5. Kent G, Minick OT, Volini FI, Orfei E. Autophagic vacuoles in human red cells. Am J Pathol. 1966;48(5):831-857.

6. Zhang J, Wu K, Xiao X, et al. Autophagy as a regulatory component of erythropoiesis. Int J Mol Sci. 2015;16(2): 4083-4094.
7. Honda S, Arakawa S, Nishida Y, Yamaguchi H, Ishii E, Shimizu S. Ulk1-mediated Atg5independent macroautophagy mediates elimination of mitochondria from embryonic reticulocytes. Nat Commun. 2014;5:4004.

8. Betin VM, Singleton BK, Parsons SF, Anstee DJ, Lane JD. Autophagy facilitates organelle clearance during differentiation of human erythroblasts: evidence for a role for ATG4 paralogs during autophagosome maturation. Autophagy. 2013;9(6):881-893.

9. Zhang J, Randall MS, Loyd MR, et al. Mitochondrial clearance is regulated by Atg7-dependent and -independent mechanisms during reticulocyte maturation. Blood. 2009;114(1):157-164.

10. Kundu M, Lindsten T, Yang CY, et al. Ulk1 plays a critical role in the autophagic clearance of mitochondria and ribosomes during reticulocyte maturation. Blood. 2008;112(4): 1493-1502.
11. Mortensen M, Ferguson DJ, Edelmann M, et al. Loss of autophagy in erythroid cells leads to defective removal of mitochondria and severe anemia in vivo. Proc Natl Acad Sci U S A. 2010;107(2):832-837.

12. Novak I, Kirkin V, McEwan DG, et al. Nix is a selective autophagy receptor for mitochondrial clearance. EMBO Rep. 2010;11 (1):45-51.

13. Sandoval H, Thiagarajan P, Dasgupta SK, et al. Essential role for Nix in autophagic maturation of erythroid cells. Nature. 2008;454 (7201):232-235

14. Schweers RL, Zhang J, Randall MS, et al. NIX is required for programmed mitochondrial clearance during reticulocyte maturation. Proc Natl Acad Sci U S A. 2007;104(49):19500-19505.

15. Matsuda N, Sato S, Shiba K, et al. PINK1 stabilized by mitochondrial depolarization recruits Parkin to damaged mitochondria 
and activates latent Parkin for mitophagy. J Cell Biol. 2010;189(2):211-221.

16. Ordureau A, Paulo JA, Zhang W, et al. Dynamics of PARKIN-dependent mitochondrial ubiquitylation in induced neurons and model systems revealed by digital snapshot proteomics. Mol Cell. 2018;70(2):211-227.

17. Narendra DP, Jin SM, Tanaka A, et al. PINK1 is selectively stabilized on impaired mitochondria to activate Parkin. PLoS Biol. 2010;8(1):e1000298.

18. Sun Y, Vashisht AA, Tchieu J, Wohlschlegel JA, Dreier L. Voltage-dependent anion channels (VDACs) recruit Parkin to defective mitochondria to promote mitochondrial autophagy. J Biol Chem. 2012;287(48): 40652-40660.

19. Geisler S, Holmstrom KM, Skujat D, et al. PINK1/Parkin-mediated mitophagy is dependent on VDAC1 and p62/SOSTM1. Nat Cell Biol. 2010;12(2):119-131.

20. Gatliff J, East D, Crosby J, et al. TSPO interacts with VDAC1 and triggers a ROS-mediated inhibition of mitochondrial quality control. Autophagy. 2014;10(12):2279-2296.

21. Shoshan-Barmatz V, Keinan N, Abu-Hamad $\mathrm{S}$, Tyomkin D, Aram L. Apoptosis is regulated by the VDAC1 N-terminal region and by VDAC oligomerization: release of cytochrome c, AIF and Smac/Diablo. Biochim Biophys Acta. 2010;1797(6-7):12811291.

22. Shimizu S, Narita M, Tsujimoto Y. Bcl-2 family proteins regulate the release of apoptogenic cytochrome $\mathrm{c}$ by the mitochondrial channel VDAC. Nature. 1999;399(6735): 483-487.

23. Rone $\mathrm{MB}$, Midzak AS, Issop $\mathrm{L}$, et al. Identification of a dynamic mitochondrial protein complex driving cholesterol import, trafficking, and metabolism to steroid hormones. Mol Endocrinol. 2012;26(11):18681882

24. Lee $\mathrm{AC}, \mathrm{Xu} \mathrm{X}$, Colombini $\mathrm{M}$. The role of pyridine dinucleotides in regulating the permeability of the mitochondrial outer membrane. J Biol Chem. 1996;271(43):2672426731.

25. Rostovtseva TK, Komarov A, Bezrukov SM, Colombini M. VDAC channels differentiate between natural metabolites and synthetic molecules. J Membr Biol. 2002;187(2):147156

26. An X, Schulz VP, Li J, et al. Global transcriptome analyses of human and murine terminal erythroid differentiation. Blood. 2014; 123(22):3466-3477

27. Gautier EF, Ducamp S, Leduc M, Salnot V, Guillonneau F, Dussiot $M$, et al. Comprehensive proteomic analysis of human erythropoiesis. Cell Rep. 2016;16(5): $1470-1484$
28. $\mathrm{Hu}$ J, Liu J, Xue F, et al. Isolation and functional characterization of human erythroblasts at distinct stages: implications fo understanding of normal and disordered erythropoiesis in vivo. Blood. 2013;121(16): 3246-3253

29. Konstantinidis DG, Pushkaran S, Johnson JF et al. Signaling and cytoskeletal requirements in erythroblast enucleation. Blood. 2012;119(25):6118-6127.

30. Wang J, Ramirez T, Ji P, Jayapal SR, Lodish HF, Murata-Hori M. Mammalian erythrob last enucleation requires PI3K-dependent cell polarization. J Cell Sci. 2012;125(Pt 2):340-349.

31. Gasko O, Danon D. Deterioration and disappearance of mitochondria during reticulocyte maturation. Exp Cell Res. 1972;75(1):159-169.

32. Simpson CF, Kling JM. The mechanism of mitochondrial extrusion from phenylhydrazine-induced reticulocytes in the circulating blood. J Cell Biol. 1968;36(1):103-109.

33. Beams HW, Kessel RG. Electron microscope and ultracentrifugation studies on the rat reticulocyte. Am J Anat. 1966;118(2):471507.

34. Bantug GR, Fischer M, Grahlert J, et al Mitochondria-endoplasmic reticulum contact sites function as immunometabolic hubs that orchestrate the rapid recall response of memory CD8+ $\mathrm{T}$ cells Immunity. 2018;48(3):542-555.e6

35. Pankiv S, Clausen TH, Lamark $\mathrm{T}$, et al p62/SOSTM1 binds directly to Atg8/LC3 to facilitate degradation of ubiquitinated protein aggregates by autophagy. J Biol Chem. 2007;282(33):24131-24145.

36. Vo MT, Smith BJ, Nicholas J, Choi YB Activation of NIX-mediated mitophagy by an interferon regulatory factor homologue of human herpesvirus. Nat Commun. 2019;10(1):3203

37. Liu X, Zhang Y, Ni M, et al. Regulation of mitochondrial biogenesis in erythropoiesis by mTORC1-mediated protein translation. Nat Cell Biol. 2017;19(6):626-638.

38. Moras M, Hattab C, Gonzalez-Menendez P et al. Downregulation of mitochondria TSPO inhibits mitophagy and reduces enucleation during human terminal erythropoiesis. Int J Mol Sci. 2020;21(23):9066.

39. Yang C, Hashimoto M, Lin OXX, Tan DQ, Suda T. Sphingosine-1-phosphate signaling modulates terminal erythroid differentiation through the regulation of mitophagy. Exp Hematol. 2019;72:47-59.

40. Gilquin B, Taillebourg E, Cherradi N, et al. The AAA+ ATPase ATAD3A controls mitochondrial dynamics at the interface of the inner and outer membranes. Mol Cell Biol. 2010;30(8):1984-1996.
41. Jin $G, X u C$, Zhang X, et al. Atad3a suppresses Pink1-dependent mitophagy to maintain homeostasis of hematopoietic progenitor cells. Nat Immunol. 2018;19(1):2940

42. Greene AW, Grenier K, Aguileta MA, et al. Mitochondrial processing peptidase regulates PINK1 processing, import and Parkin recruitment. EMBO Rep. 2012;13(4):378 385 .

43. Heo JM, Harper NJ, Paulo JA, et al. Integrated proteogenetic analysis reveals the landscape of a mitochondrial-autophagosome synapse during PARK2-dependent mitophagy. Sci Adv. 2019;5(11):eaay4624

44. Hoshino A, Wang WJ, Wada S, et al. The ADP/ATP translocase drives mitophagy independent of nucleotide exchange. Nature. 2019;575(7782):375-379.

45. Traxler EA, Thom CS, Yao Y, Paralkar V Weiss MJ. Nonspecific inhibition of erythropoiesis by short hairpin RNAs. Blood. 2018;131(24):2733-2736.

46. Lupo F, Tibaldi E, Matte A, et al. A new molecular link between defective autophagy and erythroid abnormalities in chorea-acanthocytosis. Blood. 2016;128(25):2976-2987.

47. Jagadeeswaran R, Vazquez BA, Thiruppathi $M$, et al. Pharmacological inhibition of LSD and $\mathrm{mTOR}$ reduces mitochondrial retention and associated ROS levels in the red blood cells of sickle cell disease. Exp Hematol. 2017;50:46-52.

48. Jiang $\mathrm{H}$, Yang L, Guo L, et al. Impaired mitophagy of nucleated erythroid cells leads to anemia in patients with myelodysplastic syndromes. Oxid Med Cell Longev. 2018:2018:6328051.

49. Sbardella D, Tundo GR, Campagnolo L, et al. Retention of mitochondria in mature human red blood cells as the result of autophagy impairment in Rett syndrome. Sci Rep. 2017;7(1):12297.

50. Wu L, Xu W, Xu L, Kong Q, Fang J. Mitophagy is increased during erythroid differentiation in beta-thalassemia. Int Hematol. 2017;105(2):162-173.

51. Li-Harms X, Milasta S, Lynch J, et al. Mito protective autophagy is impaired in ery throid cells of aged mtDNA-mutator mice. Blood. 2015;125(1):162-174

52. Houwerzijl EJ, Pol HW, Blom NR, van der Want JJ, de Wolf JT, Vellenga E. Erythroid precursors from patients with low-risk myelodysplasia demonstrate ultrastructural features of enhanced autophagy of mitochondria. Leukemia. 2009;23(5):886 891.

53. Koschade SE, Brandts CH. Selective autophagy in normal and malignant hematopoiesis. J Mol Biol. 2020;432(1):261 282. 